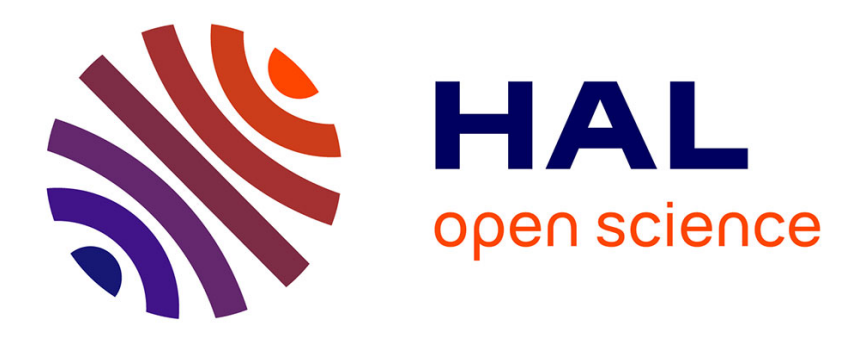

\title{
La médiation de rue comme régulation des désordres urbains
}

Éric Marlière, Séverine Fontaine

\section{To cite this version:}

Éric Marlière, Séverine Fontaine. La médiation de rue comme régulation des désordres urbains. Pensée Plurielle - Parole, pratiques et réflexions du social , 2011, nº 26 (1), 10.3917/pp.026.0013 . hal01638279

\section{HAL Id: hal-01638279 \\ https://hal.science/hal-01638279}

Submitted on 25 Nov 2017

HAL is a multi-disciplinary open access archive for the deposit and dissemination of scientific research documents, whether they are published or not. The documents may come from teaching and research institutions in France or abroad, or from public or private research centers.
L'archive ouverte pluridisciplinaire HAL, est destinée au dépôt et à la diffusion de documents scientifiques de niveau recherche, publiés ou non, émanant des établissements d'enseignement et de recherche français ou étrangers, des laboratoires publics ou privés. 


\section{LA MÉDIATION DE RUE COMME RÉGULATION DES DÉSORDRES URBAINS}

Éric Marlière, Séverine Fontaine

De Boeck Supérieur | «Pensée plurielle»

2011/1 $n^{\circ} 26$ | pages 13 à 23

ISSN 1376-0963

ISBN 9782804165192

Article disponible en ligne à l'adresse :

https://www.cairn.info/revue-pensee-plurielle-2011-1-page-13.htm

\section{Pour citer cet article :}

Éric Marlière, Séverine Fontaine «a médiation de rue comme régulation des désordres urbains », Pensée plurielle 2011/1 (n²6), p. 13-23.

DOI 10.3917/pp.026.0013

Distribution électronique Cairn.info pour De Boeck Supérieur.

(C) De Boeck Supérieur. Tous droits réservés pour tous pays.

La reproduction ou représentation de cet article, notamment par photocopie, n'est autorisée que dans les limites des conditions générales d'utilisation du site ou, le cas échéant, des conditions générales de la licence souscrite par votre établissement. Toute autre reproduction ou représentation, en tout ou partie, sous quelque forme et de quelque manière que ce soit, est interdite sauf accord préalable et écrit de l'éditeur, en dehors des cas prévus par la législation en vigueur en France. Il est précisé que son stockage dans une base de données est également interdit. 


\title{
La médiation de rue comme régulation des désordres urbains
}

\author{
ÉRIC MARLIÈRE ET SÉVERINE FONTAINE 1
}

\begin{abstract}
Résumé : Les ruptures entre les jeunes dits « des cités 》 et les élus locaux se sont opérées progressivement depuis les années 1980. Elles se sont accentuées brutalement avec les émeutes de novembre 2005, comme le montrent les récentes échauffourées entre jeunes et police dans les cités populaires urbaines. Quelle que soit la culture politique de la commune, les élus locaux ont de plus en plus de mal à conserver de la crédibilité à l'égard d'une frange croissante de la jeunesse des quartiers populaires, celle qui se situe en marge. C'est le cas d'une municipalité " gauche » de la banlieue de Paris étudiée entre 2007 et 2008 qui voit une augmentation graduelle des tensions entre jeunes et institutions. Cette situation que l'on pourrait qualifier d' " explosive " amène les édiles municipaux à concevoir (avant les émeutes de 2005) un dispositif de médiation de rue composé d'adultes originaires des cités HLM qui composent la ville.
\end{abstract}

Mots clés : médiation de rue, dispositif expérimental, élus municipaux, "banlieue rouge ", recompositions politiques, politique de la ville, jeunesse populaire, prévention, "culture de rue », conflits, violences urbaines, paix sociale, négociations.

Depuis bientôt trente ans, la jeunesse des « quartiers sensibles » est devenue un nouvel acteur social. Les émeutes urbaines de 2005 ont donné à celle-ci une dimension politique nationale marquant un véritable tournant dans le paysage politique français. Si, dans les années 1980, avec la fermeture des usines et la décomposition du monde ouvrier, certains chercheurs se posaient déjà la question de l'avenir des enfants d'ouvriers et/ou d'immigrés (Dubet, 1987 ; Bouamama, 1994), vingt ans plus tard, la situation s'est dégradée au point que nombre de sociologues pensent que nous sommes passés de la génération « galère » et « marche pour l'égalité » dans les années 1980 à une

\footnotetext{
${ }^{1}$ Éric MARLIÈRE, maître de conférences à l'université Lille 3, chercheur au CeRIES EA 3589 et Séverine FONTAINE, doctorante en sociologie sous la direction de Serge PAUGAM, ERIS (Équipe de Recherche sur les Inégalités Sociales), EHSS/ENS.
} 
“ génération d'émeutiers » deux décennies plus tard (Beaud \& Masclet, 2006). Les recherches empiriques récentes attestent d'un sentiment vécu d'abandon et de désespoir conjugué à celui d'injustice fortement ancré pour la plupart des jeunes rencontrés dans les cités populaires, quels que soit leur âge, leurs parcours scolaire et professionnel (Marlière, 2008a).

La récurrence du phénomène de "violences urbaines » témoigne d'une cassure politique et symbolique grandissante entre les élites politiques nationales et locales, d'une part, et les habitants des quartiers populaires, notamment les plus jeunes, d'autre part. Au-delà des travaux qui soulignent l'importance de l'abstention (Braconnier \& Domargen, 2005) et de la désaffiliation (Bacqué \& Syntomer, 2001), il est essentiel de s'interroger sur les effets des mutations économiques qui évoluent dans les « quartiers sensibles ». Pour pallier les nombreux échecs et insuffisances des politiques publiques à l'égard des nouvelles générations en France depuis plus de trente ans maintenant (Loncle, 2010), des services de médiation voient le jour dans un certain nombre de banlieues populaires. Ces nouveaux services, dépendant des nouvelles politiques de prévention, sont la plupart du temps constitués de jeunes adultes, dont la compétence principale est la maîtrise des codes de la " culture de rue » mais aussi une connaissance des réseaux sociaux locaux. En effet, une partie de ces jeunes adultes rencontrés sur notre terrain dispose d'un capital d' " autochtonie » indispensable à la régulation des désordres juvéniles urbains (Rétière, 2003). De ce capital, ces médiateurs formés à l' " univers de la rue » sont en mesure d'extraire des "savoir-faire ", transformés en pratiques professionnelles leur permettant de gérer les conflits, voire de pallier un grand nombre de débordements.

Cet article repose sur l'observation de près de deux ans d'une ville de la " ceinture rouge " parisienne aux prises avec la décomposition du monde ouvrier et de son système social. Les élus municipaux peinent à trouver les solutions permettant de faire face à cette jeunesse « turbulente " (Boucher, 2003) qui n'a rien à perdre. Le service de médiation, composé de trentenaires avancés des quartiers populaires locaux, montre toute l'ambivalence d'un dispositif de régulation des désordres urbains, devant cette jeunesse populaire en difficulté dont la présence, la " visibilité » et plus encore les nuisances associées à celles-ci troublent dans certains cas l'ordre public. Les élus locaux doivent faire face à l'urgence (électorale) en gérant les attitudes et comportements pathogènes de cette jeunesse populaire en proie à de grandes difficultés sociales. Les élus de la majorité municipale d'un ancien parti ouvrier cumulent les injonctions contradictoires qui consistent à rassurer en partie les « classes populaires encore stables " (notamment les personnes âgées). En fait, les politiques locaux peinent non seulement à gérer cette jeunesse en marge aujourd'hui, mais pire encore, ils ne sont plus en mesure d'en anticiper les réactions.

\section{La mise en place d'une expérimentation locale : un dispositif de « médiation de rue »}

Traditionnellement, les élus du parti ouvrier, cultivant ou jouissant d'une certaine proximité (sociale, culturelle, spatiale), occupent d'un point de vue sociologique des fonctions de médiation dans la cité. La proximité sociale et 
culturelle de ces élus d'origine ouvrière se faisait naturellement avec la population. C'est pourquoi la mise en place d'un tel dispositif est en quelque sorte un impensé, ou plus exactement la reconnaissance implicite et honteuse d'un schisme qu'il convient de cacher le plus possible. Pour être plus précis, la mise en œuvre d'une politique telle que celle-ci marque trois aspects essentiels : 1) Tout d'abord, il s'agit pour les politiques locaux d'un tournant dans la gestion publique de la ville. 2) La création d'un tel service au sein même de la structure municipale est, tour à tour, la reconnaissance pragmatique d'une sévère fracture entre des élus populaires et une partie plus ou moins importante des jeunesses de la ville. 3) C'est aussi le constat de l'échec d'une politique qui a longtemps investi et misé sur cette frange de la population. Réfractaires pour des raisons idéologiques et dogmatiques à la création d'un service de police municipale, les élus municipaux mettent en place un service de médiation de jeunes adultes issus des quartiers afin de pouvoir renouer au mieux les contacts avec les jeunes estimés « difficiles ». La tâche ultime étant d'instrumentaliser un tel outil de proximité, véritable vecteur de communication et de dialogue, et donc de régulation - au sens de la tranquillité publique - des comportements des plus jeunes concitoyens.

Avant de détailler davantage ce dispositif, revenons très rapidement sur le sens même, et surtout sur la manière de faire de la médiation. La médiation est un terme très générique, bien moins explicite qu'il n'y paraît. Son usage galvaudé ces dernières années nous laisse penser qu'il recouvre une homogénéité de pratiques, tandis que la réalité est bien différente (Milburn, 2002). Derrière cet actuel engouement, se cachent en réalité plusieurs conceptions qui se juxtaposent les unes aux autres pour former un tout hétérogène au sein duquel différentes formes coexistent (médiation pénale, familiale, culturelle, de rue...). Parmi toutes ces formes possibles, la médiation a comme dénominateur commun d'être une pratique fondée sur l'art du langage. Celle-ci permet de créer ou de recréer du lien entre des personnes en conflit et implique l'intervention d'un tiers neutre, impartial et indépendant, le médiateur. Cela étant dit, nous pourrions préciser que, dans le contexte des « quartiers sensibles », le travail de médiateur est l'ultime recours des institutions municipales pour pouvoir contenir les débordements de plus en plus récurrents d'une partie des adolescents des quartiers populaires. En effet, le travail de médiateur de rue, souvent exercé par des « pacificateurs indigènes » (Boucher, 2010), se concrétise et se pérennise dans un contexte où le travailleur social diplômé d'État ne peut intervenir.

Ce qu'il faut comprendre, c'est qu'il existe deux manières bien distinctes de faire de la médiation. La première consiste à faire intervenir un tiers extérieur, étranger à la situation, dont l'impartialité est présupposée plus simple de par sa non-implication dans l'affaire concernée. L'autre manière, radicalement différente, nécessite le recours à un tiers intérieur, quelqu'un qui connaît les tenants et les aboutissants du conflit ou encore de telle dégradation de situation ; en un mot, un tiers qui maîtrise l'historique (c'est-à-dire aussi bien l'histoire locale et/ou familiale que l'imbrication relationnelle forte dans laquelle s'enracinent les ressorts des conflits, y compris territorialement dans l'espace de la ville comme des conséquences que ces ressorts territoriaux font reposer sur le fragile équilibre pacifique communal, etc.). Enfin, le recours à une forme 
de médiation interne induit implicitement un rapport de confiance étroit, puisque le médiateur appartient « de manière pleine et entière » à la communauté, de laquelle il est même désigné pour occuper une fonction à part. Son élection a pour présupposé sa capacité à savoir s'extirper de sa condition « primaire » de simple habitant de quartier et de façon désintéressée. C'est aussi là une des difficultés de cette mission : savoir se montrer impartial, en laissant de côté préjugés, intérêts personnels et vieilles inimitiés, pour se montrer équitable et intègre et, par là, être reconnu légitimement comme tiers à part entière pour réguler les conflits et autres problèmes de violence.

La ville sur laquelle reposent nos observations a très clairement opté pour cette seconde vision dans la mesure où les processus de ségrégation en cours (Preteceille, 2009) amènent les habitants et les jeunes à se montrer de plus en plus hostiles envers les institutions et intervenants « étrangers ». Dans ce cas, la commune étudiée s'est inspirée des expérimentations d'autres localités pour se constituer un système propre tout en refusant énergiquement, au moins officiellement, tout système évoquant celui des « grands frères ". Comme nous l'avons rappelé au tout début de notre exposé, cette ancienne commune ouvrière a conservé une forte tradition populaire et cultive également une vigilance et une méfiance toute particulière à l'égard de tout ce qui lui est extérieur. Bien sûr, cela procède d'un ensemble de facteurs divers, dont la culture ouvrière est un élément constitutif, mais cela tient également à son histoire politique symptomatique qu'il n'est pas possible de dévoiler davantage sans craindre de rendre notre commune identifiable et, avec elle, ses acteurs.

Les médiateurs choisis habitent donc les quartiers de la ville. La proximité géographique et sociale est une position confortable pour beaucoup de médiateurs. Pourtant, cette position est très critiquée par d'autres professionnels tels les animateurs ou les éducateurs spécialisés. Ces derniers déconsidèrent publiquement les tâches des médiateurs (non-diplômés d'une école du travail social et d'une « compétence » identifiable, etc.) car ils les estiment inutiles, ambigus et sans doute peu professionnels. En effet, il n'est pas aisé pour un médiateur d'être en même temps « dedans » tout en étant « indépendant et impartial ». Cela suppose de pouvoir « naviguer » entre plusieurs univers, parfois antinomiques, d'être en mesure d' « enfiler plusieurs casquettes » et de développer des compétences informelles liés à l'expérience de la rue et d'un capital d' « autochtonie ». Ceci confère à la mission du médiateur une dimension éprouvante sur le long terme. En outre, cette situation particulière suppose également que le professionnel indique à chaque fois l'étiquette selon laquelle le médiateur intervient (simple habitant, médiateur, frère d'untel, etc.). Car si nous avons précisé qu'il s'agissait d'une expérimentation novatrice, nous n'avons pas ajouté que celle-ci a été menée de manière discontinue durant près de deux ans avant de pouvoir devenir un dispositif pérenne (c'est-à-dire s'inscrivant de manière régulière).

Dans ce contexte bien précis, la médiation qui se dessine et commence à prendre forme revêt un caractère éminemment novateur et expérimental, aussi bien dans ses missions que dans ses manières de faire et d'être. Les recrutements essentiellement locaux sont aussi complexes que peu académiques. En effet, ces adultes ne sont pas diplômés d'État d'une école du travail social et 
révèlent dans une certaine mesure le processus d'une déqualification du travail social, notamment dans les emplois subalternes ou les tâches difficiles (Autès, 1999). Les médiateurs sont pour une part non qualifiés, et leurs compétences réelles ne reposent pas sur la détention d'éventuels diplômes, mais bien davantage sur le fait de connaître les problématiques liées à la jeunesse populaire et à l'expérience « de la rue ». C'est dans ce contexte que réside la véritable compétence des médiateurs dans la manière de gérer les conflits juvéniles et de maintenir un certain ordre public. Si les médiateurs ont donc une « carte à jouer » au niveau de l'institution, c'est bien celle de leur expérience personnelle à repérer les problèmes ou encore, de façon plus subtile, à repérer les enjeux de nature conflictuelle qui peuvent amener à des violences. II s'agit d'avoir un sens aiguisé de l'observation, joint à une connaissance fine de chacun des quartiers auxquels les médiateurs sont attachés, permettant une lecture analytique rapide et intuitive des situations. Cette lecture fine et précise permet de connaître rapidement l'état des relations entre groupes de jeunes occupant l'espace public, notamment lorsque ceux-là s'inscrivent de manière prolongée et/ou nocturne. Elle permet aussi d'anticiper, au regard des différentes occupations de l'espace public, les tensions entre ces mêmes groupes, rassemblés autour d'activités de nature diverse (sportives, amicales, voire délictuelles...).

Chacun des médiateurs recrutés l'a été en fonction de caractéristiques personnelles (histoires de vie singulières, inscription locale et associative forte, etc.) et relationnelles propres (réseaux d'appartenance familiale et/ou amicale), dont chacune déterminait des connaissances spécifiques, mais complémentaires, associées à des micro-découpages particuliers du territoire, dont la seule coordination de l'ensemble de l'équipe permet d'avoir une lecture globale, le tout reposant sur une solide culture de la rue. Ces micro-découpages invisibles du territoire sont le fruit des différentes logiques segmentaires qui traversent la ville (différenciation spatiale, sociale, ethnique, générationnelle, religieuse, etc.) et qui sont aussi produites par la rue elle-même, dont l'imposition rigide des codes est rendue possible par la menace et/ou l'usage de la violence qui l'accompagne de façon systématique (Bourgois, 2001).

Si les objectifs visés d'un tel dispositif sont clairement le maintien pragmatique de l'ordre public dans la rue et ce, quels que soient les moyens utilisés, la médiation s'apparente à une forme d'achat de la paix sociale. En effet, les expériences passées des « grands frères » ont laissé des traces et, dans certains cas, des traumatismes pour bon nombre d'élus et de responsables associatifs. C'est pourquoi il est question dorénavant de donner l'illusion aussi bien auprès des habitants que des Institutions (Préfecture, Police, Conseil général...) que la Ville est en capacité de gérer et ne se laisse plus malmener par des groupes violents de pression. Toutefois, il faut reconnaître que là aussi le jeu des politiques locaux est complexe puisque les débordements sont aussi une manière, pour eux, de dénoncer une politique nationale à laquelle ils s'opposent, au moins publiquement, de manière vigoureuse et dont les effets dévastateurs des violences urbaines permettent de se repositionner politiquement tout en récupérant des subsides de l'État, dont ils n'ont de cesse de dénoncer et de combattre le désengagement chronique. 


\section{Politique préventive au détriment d'une action sociale pour la jeunesse en difficulté}

Si les processus de civilisation sont toujours en cours et s'illustrent, pour ce qui nous intéresse ici, par une moins grande tolérance vis-à-vis de la violence et des incivilités dans l'opinion publique (Mucchielli, 2009), on comprend mieux pourquoi la « question des banlieues " apparaît de plus en plus problématique pour les élus de tous bords. Les attentes électorales en matière de sécurité incitent les responsables politiques à chercher des solutions rapides au détriment d'une action publique sur le long terme visant à l'amélioration des conditions de vie des habitants des quartiers populaires. Les mutations évoquées au début de notre article se révèlent également dans les recompositions de l'action politique où la problématique de gestion des "risques sociaux " prend le pas sur la " question sociale ». Si nous sommes entrés dans l'ère de la société dite post-industrielle depuis plus de trente ans maintenant, les effets les plus manifestes s'illustrent dans les mutations qui sont en cours au niveau des politiques de sécurité. La dépolitisation de la «question sociale » laisse place à une re-politisation des questions sécuritaires (Boucher, 2004) et d'une nouvelle gestion des risques urbains par une politique plus répressive (Bonelli, 2008) pour un ensemble conséquent d'anciennes communes ouvrières toujours communistes (Tissot, 2007). Et d'un point de vue local, les anciens représentants politiques de la classe ouvrière sont aujourd'hui contraints de s'adapter aux nouvelles " demandes de sécurité " (Lagrange, 2003), quitte à se révéler plus distants de leur électorat populaire. La décomposition des "banlieues rouges » appelle de nouvelles stratégies de la part des élus « ouvriers » qui sont confrontés à des choix pragmatiques en raison de l'urgence politique électorale. La politique « politicienne » actuelle incite les élus à favoriser la venue de classes moyennes stables (Bacqué \& Fol, 1997) et à maintenir une gestion aléatoire de populations ouvrières paupérisées (enclines encore à voter à gauche). Cette stratégie politique ne peut aboutir qu'en régulant une jeunesse en voie de marginalisation sociale et de moins en moins contrôlable dans l'espace public.

Cette situation montre toute l'ambiguïté des modes d'intervention publique dans les quartiers populaires depuis presque trois décennies. Les politiques de la ville ne traduisent rien d'autre finalement que la volonté de maintenir coûte que coûte la cohésion sociale et la tranquillité publique au détriment d'une amélioration sensible des conditions de vie des habitants des cités populaires urbaines paupérisées par la désindustrialisation et ses effets (précarité, chômage, etc.). Les élus de droite comme de gauche ne sont plus en mesure de trouver des leviers institutionnels indispensables pour permettre une émancipation sociale et économique des classes populaires et notamment de sa jeunesse urbaine en grandes difficultés. En d'autres termes, les élus locaux sont de plus en plus confinés au seul traitement des tâches urgentes qui consistent soit à résoudre tant bien que mal les problèmes liés à la marginalité urbaine ou à la régulation d'émeutes sporadiques mais de plus en plus récurrentes dans un certain nombre de quartiers populaires. Ce constat manifeste une réelle impuissance des politiques publiques à redynamiser le tissu économique et social local. C'est sans aucun doute également le constat d'un échec des 
politiques de la ville dans le domaine d'animation des quartiers populaires en matière de précarité, de disqualification sociale ou de régulation des désordres urbains (Donzelot \& Estebe, 1994). C'est dans ce contexte, par exemple, que certains sociologues constatent en observant les missions locales pendant plus de vingt ans que celles-ci ne voient plus les jeunes des quartiers populaires comme de futurs travailleurs, mais plutôt comme des exclus durables et sans doute définitifs du marché de l'emploi (Tourillhès, 2008).

Le dispositif de médiation de rue illustre en quelque sorte le renoncement à l'amélioration de la situation des classes populaires et à une volonté d'insérer professionnellement les « jeunes de cité » contrairement aux travailleurs sociaux classiques qui essaient de transformer les institutions et l'environnement dans le souci de l'émancipation sociale des usagers (Boucher, 2004b). Le dispositif de médiation traduit une logique de gestion de la tranquillité publique, dont le volontarisme municipal affiché cache en réalité un subtil achat de la paix sociale, tranquillement négociée à l'ombre des violences urbaines. Ces violences, qui ont, par ailleurs, comme toile de fond la souffrance et le désespoir bien réels d'une partie de la jeunesse populaire confinée aux marges d'une société post-industrielle dans laquelle elle peine de plus en plus à s'insérer, sont en réalité entièrement instrumentalisées par les deux bouts de la chaîne (politiques ou groupes de juvéniles «instables »). Cette situation conduit désormais à l'impuissance des pouvoirs publics à modifier le destin tragique d'une partie des classes populaires urbaines déprolétarisées mais aussi à l'échec d'une négociation avec les jeunes évoluant dans les « quartiers sensibles » de plus en plus suspicieux à l'égard des politiques et des institutions (Marlière, 2010).

\section{Le « chantage à l'émeute »: un moyen de négociation efficace pour certains médiateurs originaires des quartiers impopulaires}

Confrontés à une situation sociale critique de plus en plus "explosive ", les élus n'ont pas d'autres recours actuellement que de faire appel à des médiateurs de rue qui bénéficient de certaines compétences pour apaiser les tensions permanentes ${ }^{2}$. Conscients de ce pouvoir infra-politique, certains médiateurs ayant intériorisé le cynisme de certains de leurs employeurs n'hésitent pas, pour leur profit personnel ou pour l'intérêt du quartier, à utiliser ce nouveau pouvoir en faisant « pression » sur les élus. Autrement dit, nos observations de terrain montrent que ce dispositif - dans un contexte que l'on peut qualifier de "sulfureux »- peut amener à terme à des relations ambiguës entre élus et médiateurs. Ainsi, nous avons pu noter l'existence de négociations informelles et surtout secrètes entre médiateurs peu scrupuleux du «bien commun » et élus en quête de reconnaissance et de légitimité, voire de reconquête politique. Bien entendu, ces négociations ont fait preuve de modes

\footnotetext{
${ }^{2}$ La plupart des municipalités disposent d'un service de police municipal sans pour autant que le travail de médiation avec les jeunes soit plus efficace. Bien au contraire, la nature des rapports entre les jeunes et la police est plus que conflictuelle aujourd'hui.
} 
de communication aussi subtils que discrets, ayant été élaborés afin que tout ceci reste invisible aux yeux des électeurs (pour les élus) mais aussi des jeunes (instrumentalisés par les médiateurs). Bien sûr, cet achat de la paix sociale par le biais de cadeaux en faveur de certains médiateurs « charismatiques » doit se concrétiser en bout de course par des améliorations aussi bien qualitatives que quantitatives des violences urbaines locales. Loin d'être apaisées, les incivilités que l'on peut définir comme urbaines se résolvent à des fins purement «politiciennes » d'élus locaux soucieux de reconquérir électoralement des territoires, ou au pire de se prolonger sur l'échiquier local en rassurant un électorat déboussolé par les mutations en cours.

Nos observations de terrain montrent que cette disposition peut conduire à terme à des controverses, car les stratégies locales pour le maintien de la tranquillité publique débouchent parfois sur des négociations politiques avec des médiateurs, placés en concurrence avec des groupes juvéniles susceptibles d'être violents et donc d'en limiter leur possibilité de créer du désordre. Ces médiateurs se découvrent alors de nouvelles potentialités (reconnues par l'institution qui les avait dénigrées jusqu'ici), notamment celle d'une possible nuisance au niveau de leur territoire local d'origine. Ce pouvoir de nuisance - le « chantage à l'émeute » et/ou aux désordres urbains - se concrétise rapidement en rapport de force avec les élus en place. Ainsi, la peur des violences urbaines, amplifiée par les émeutes de novembre 2005, favorise les possibilités de négociations des médiateurs avec les élus en désir permanent de reconquête de légitimité. Ces arrangements médiateurs/élus municipaux sont aussi la marque de la négociation fine de certains médiateurs avec quelques groupes de jeunes reconnus pour leur violence, et avec lesquels les élus comme les institutions peinent à communiquer. Cette situation pour le moins équivoque incite les élus à composer avec les médiateurs quitte à passer outre les manières plus habituelles de régler les désordres urbains.

C'est pourquoi certains médiateurs sont prêts à offrir aux élus locaux également peu scrupuleux leurs « bons (mais pas loyaux) services ». Cela va d'une gestion subtile des revendications agressives des « jeunes de cité " à la possibilité de circulation des élus dans les «quartiers chauds » de la ville en passant par le « déminage » des situations émeutières ou de violences qualifiées d'urbaines. Les médiateurs, au-delà du salaire que leur procure la fonction, arrivent à obtenir des avantages en contreparties individuelles (prêts immobiliers à taux négociés, rétributions financières diverses, négociation de postes pour des conjoints, acquisition d'un logement social en temps record sur un marché saturé, etc.) ou collectives (subvention pour des associations de quartier dont ils sont membres). Le « chantage à l'émeute » est donc un outil de persuasion efficace pour des médiateurs conscients que l'électorat est de plus en plus exigeant en matière d'efficacité sécuritaire.

Ces négociations « infra-politiques » souvent tacites renforcent le pouvoir des médiateurs dans les quartiers où ils officient, dès lors qu'ils communiquent sur les profits réalisés au nom du collectif (subvention pour les associations de quartier), seul vecteur de prestige symbolique. Cela permet une gestion des violences locales d'une efficacité plus grande, mais qui impose pour le groupe promu (les médiateurs en l'occurrence) d'endosser, ne serait-ce qu'un temps, le symbole du pouvoir politique municipal. Appréhendée comme une 
compromission pour beaucoup de " jeunes de cité ", la situation des médiateurs est en totale contradiction avec l'" univers de la rue ». L'affiliation affichée provisoirement entre élus et médiateurs de rue issus des " quartiers " démontre la crainte politique, sociale et symbolique que suscite la jeunesse des « quartiers sensibles » aujourd'hui. Ces relations " contre nature » (élus/ anciens « jeunes de cité » devenus médiateurs) montrent en partie la déliquescence qui affecte une partie des institutions locales.

N'ayant « rien à perdre » et peu représentés politiquement, les « jeunes de cité " semblent constituer une menace grandissante. Issus des mêmes quartiers et ayant la même origine sociale, les médiateurs en quête de réussite économique et de reconnaissance sociale ont bien compris les enjeux politiques locaux. En effet, les médiateurs, reconnus par les jeunes, ont les moyens d'atténuer les désordres ou au contraire de fomenter des violences. Ils sont surtout au contact des élus soucieux de maintenir un ordre social considéré comme de plus en plus injuste par les jeunes et habitants des quartiers populaires (Masclet, 2003 ; Marlière, 2008b). Cette compromission permet aux médiateurs de bénéficier d'une aura particulière en raison du pouvoir qu'ils ont hérité de leur capacité à gérer l'« ingérable ». Face à des élus municipaux déconcertés par une problématique de plus en plus explosive de la "question sociale " qu'ils ne sont pas parvenus à résoudre dans les décennies précédentes, les médiateurs de rue bénéficient d'une conjoncture paradoxalement pour eux à savoir la manifestation de plus en plus récurrente des violences urbaines. En d'autres termes, la pérennisation de ce pouvoir « infra-politique » illustré par la fonction des médiateurs, coincés entre des habitants des cités qu'ils connaissent depuis leur enfance et leurs employeurs que sont les responsables politiques locaux, dépend à la fois de la «question sociale » et de la problématique juvénile de plus en plus fragile et incertaine ${ }^{3}$. Ce qui explique pourquoi certains médiateurs profitent de la soufrière que constitue la jeunesse locale désabusée et exclue politiquement en développant des rapports clientélistes " secrets " avec les élus. C'est dans un contexte social dégradé où se développe une peur collective de l'émeute de plus en plus récurrente (Bertho, 2009) que certains médiateurs peu scrupuleux favorisent la stratégie du « chantage à l'émeute » en raison d'une impuissance des élus locaux à réguler efficacement les violences urbaines et surtout à atténuer les problèmes sociaux.

\section{Conclusion}

Les violences qualifiées médiatiquement d'urbaines incitent les élus à élaborer des expérimentations nouvelles en raison des configurations locales difficiles, comme le dispositif de médiation municipal étudié dans cet article. Ce dispositif illustre en somme un jeu complexe entre élus, médiateurs et jeunes

\footnotetext{
${ }^{3}$ À l'époque de la rédaction de cet article, le service de médiation a été dissous. La situation a donc changé sensiblement depuis notre première étude (Fontaine \& Marlière, 2009). Une autre équipe de médiation composée, depuis, d'individus extérieurs ne semble pas davantage avoir l'approbation des élus puisque la municipalité vient de mettre un terme à sa mission (octobre 2010). II semblerait que les élus sont à la recherche d'un compromis acceptable entre locaux et extérieurs pour le prochain dispositif.
} 
potentiellement violents et/ou émeutiers. Les médiateurs de rue conscients du pouvoir que procure l'atmosphère incendiaire qui se développe dans les quartiers « impopulaires » disposent de nouveaux pouvoirs qui pourraient échapper à moyen terme aux élus en place. Certains d'entre eux négocient directement (en catimini cependant) avec certains élus locaux influents, afin d'obtenir des avantages en échange de services. Pour les élus, l'intérêt pour l'insécurité (Robert, 2002) est devenu prépondérant dans leur manière de communiquer et de gouverner la commune. Cette préoccupation se révèle avec acuité lorsqu'il est question d'incivilités urbaines, de désordres juvéniles, d'émeutes, de violences et de délinquances. La tentation qui consiste à développer des logiques clientélistes devient dès lors plus forte pour nombre de médiateurs tentant de monnayer leur présence dans leurs « quartiers sensibles ». En effet, ces adultes devenus des "références " par leur passé ou leur charisme ont intégré le fait que la peur collective suscitée par les violences urbaines constituait une arme stratégique redoutable auprès d'élus soucieux de maîtriser l'ensemble des désordres sur le territoire communal. Ces élus municipaux, à défaut de pouvoir agir concrètement sur l'avenir social de la jeunesse des " quartiers » confrontée à la forte désindustrialisation et à la reconfiguration d'un marché du travail de plus en plus impitoyable ${ }^{4}$, entretiennent leur position en agissant sur les conséquences visibles des problèmes sociaux en maintenant l'ordre public, en luttant « ouvertement » contre les fauteurs de troubles ou en négociant avec des médiateurs de rue pour qu'ils puissent directement atténuer concrètement les tensions juvéniles locales.

\author{
Éric MARLIÈRE \\ IUT B - Université Lille 3 \\ 35 rue Sainte-Barbe \\ BP 460 - 59208 Tourcoing Cédex \\ Tél. : 03.20.76.25.42 \\ eric.marliere@univ-lille3.fr \\ Séverine FONTAINE \\ Doctorante en sociologie \\ ERIS/ENS/EHESS \\ Chef de projet CLS / Collectivité territoriale \\ Tél. : 01.47.60.83.78 \\ fontaine.severine@free.fr
}

\title{
Bibliographie
}

AUTÈS M., 1999, Les paradoxes du travail social. Paris, Dunod.

BACQUÉ M.-H. \& FOLS S., 1997, Le devenir des banlieues rouges. Paris, L'Harmattan.

BEAUD S. \& MASCLET O., 2006, « Des "marcheurs de 1983" aux "émeutiers" de 2005. Deux générations sociales d'enfants d'immigrés ", Les Annales HSS, pp.809-843.

BERTHO A., 2009, Le temps des émeutes. Paris, Bayard.

BONELLI L., 2008, La France a peur. Histoire sociale de l'insécurité. Paris, La Découverte.

${ }^{4}$ Voir le rapport accablant sur le chômage des jeunes dans les quartiers populaires. 
BOUAMAMA S, 1994, Dix ans de marche des beurs. Chronique d'un mouvement avorté. Paris, Desclée de Brouwer.

BOUCHER M., 2010, Les internés du ghetto. Ethnographie des confrontations violentes dans une cité impopulaire. Paris, L'Harmattan.

BOUCHER M., 2004a, Repolitiser l'insécurité. Sociographie d'une ville ouvrière. Paris, L'Harmattan.

BOUCHER H., 2004b, « Travail social, intervention sociale et pacification sociale : d'un projet d'intégration et de contrôle à une action d'émancipation ", Recherches sociologiques et anthropologiques, XXXV, 3, 2004, pp. 137-151.

BOUCHER M., 2003, Turbulences, contrôle et régulation sociale. Paris, L'Harmattan.

BRACONNIER C. \& DOMARGEN J.-Y., 2005, La démocratie de l'abstention. Paris, Folio.

CASTEL R., 1995, La métamorphose de la question sociale. Une chronique du salariat. Paris, Fayard.

DONZELOT J. \& ESTĖBE P., 1994, L'État animateur. Essai sur la politique de la ville. Paris, L'Harmattan.

DUBET F., 1987, La galère : jeunes en survie. Paris, Arthème Fayard.

FONTAINE S. \& MARLIÈRE E., 2009, « Médiation de rue et logiques politiques municipales », VEI Diversité, 158, pp. 202-207.

LAGRANGE H., 2003, Demandes de sécurité. France, Europe, États-Unis. Paris, Le Seuil. LONCLE P., 2010, Politiques de jeunesses. Les défis majeurs de l'intégration. Paris, P.U.R. MARLIÈRE E., 2010, « Le sentiment d'injustice comme construction d'une identité collective chez les "héritiers des quartiers populaires" ", in Boucher M., éd., Penser les questions sociales et culturelles : quels enjeux pour l'intervention sociale ? Paris, L'Harmattan, pp. 225239.

MARLIÈRE E., 2009, « Jeunes des banlieues et émeutes urbaines », in Roudet B., éd., Regard sur les jeunes en France. Québec, P.U.L., pp. 127-145.

MARLIÈRE E., 2008a, La France nous a lâchés ! Le sentiment d'injustice chez les jeunes de cité. Paris, Fayard.

MARLIÈRE E., 2008b, « Des "ouvriers communistes" aux "jeunes des cités" : histoire croisée de la non-reconstruction d'une demande sociale ", in Anquetin V., Freyermuth A., éds, La figure de l'" habitant ». Sociologie politique de la « demande sociale ». Rennes, Presses Universitaires de Rennes, pp. 115-129.

MARLIĖRE E., 2006, «Changement, incertitude et transition sécuritaire : vers un nouveau mangement de la question sociale ? ", Management et sciences sociales, Paris, L'Harmattan, pp. 183-193.

MASCLET O., 2003, La gauche et les cités. Enquête sur un rendez-vous manqué. Paris, La Dispute.

MILBURN P, 2002, La médiation. Expériences et compétences. Paris, La Découverte.

MUCCHIELLI L., 2008, « Une société plus violente ? Une analyse socio-historique des violences interpersonnelles en France, des années 1970 à nos jours ", Déviance et Société, 32, 2, pp. 115-147.

PRETECEILLE E., 2009, « La ségrégation ethno-raciale a-t-elle augmentée dans la métropole parisienne ? ", Revue Française de sociologie, 50, 3, pp. 489-519.

RETIÈRE J.-N., 2003, « Autour de l'autochtonie. Réflexions sur la notion de capital social populaire », Politix, vol. 16, 63, pp. 121-243.

ROBERT Ph, 2002, L'insécurité en France. Paris, La Découverte et Syros.

TISSOT S., 2007, L'État et les quartiers. Genèse d'une catégorie de l'action publique. Paris, Le Seuil.

TOURRILHÈS C., 2008, Construction sociale d'une jeunesse en difficultés. Innovations et ruptures. Paris, L'Harmattan. 